\title{
Achieving High Breast Cancer Survival for Women in Rural and Remote Areas
}

\author{
Albert Hoang Nguyen Ho-Huynh ${ }^{1 *}$, Timothy J Elston ${ }^{2}$, Ronny K Gunnarsson ${ }^{3,4}$, \\ Alan de Costa ${ }^{5}$
}

\begin{abstract}
Background: Significant improvements in breast cancer survival have been made in the past few decades in many developed countries including Australia with a five-year relative survival of $90 \%$. The aim of the present study is to obtain a brief estimate of the relative importance of demographic factors such as rurality, socio-economic standard and ethnicity versus traditional risk factors for women diagnosed with breast cancer in Far North Queensland, Australia. Methods: This was a retrospective longitudinal study of all women diagnosed with their first episode of breast cancer in 1999-2013 in Far North Queensland, Australia. Cox proportional hazards regression analysis was used to identify factors independently associated with mortality for women with any type of breast cancer (in situ or invasive) and for women with invasive cancer. Life tables were used to assess five and ten-year absolute survival. Standard linear regression and binary logistic regression were used to identify any association between demographic factors and late presentation. Results: Five and ten-year absolute survival was 0.90 and 0.86 respectively. Aboriginal and Torres Strait Islander status, remoteness of area of residence, and socioeconomic status were not associated with more advanced disease at presentation or increased risk of breast cancer death. Only traditional risk factors such as increased tumour size, absence of progesterone receptor, high tumour grade and presence of metastasis in axillary lymph nodes were associated with increased risk of breast cancer death. Conclusion: The effect of the classical risk factors on breast cancer mortality outweighs the effects of demographic factors. The fact that ethnicity, remoteness and socioeconomic status is not associated with late presentation or breast cancer death suggests that given appropriate resources it may be possible to close the gap of inequalities in breast cancer.
\end{abstract}

Keywords: Breast neoplasms- risk factors- mortality- epidemiology- Australia

Asian Pac J Cancer Prev, 23 (1), 101-107

\section{Introduction}

Significant improvements in breast cancer survival have been made in the past few decades in many developed countries including Australia with a five-year relative survival of $90 \%$ (Ghoncheh et al., 2016). This is largely attributed to earlier detection, improved management and consistent approaches to treatment for breast cancer. (Youlden et al., 2012). In the US living in rural areas may not be associated with a poorer prognosis (McLafferty and Wang, 2009). However, in Australia there has been poor outcomes for women living in rural and remote areas compared to major cities, women with lower socioeconomic status and Aboriginal and Torres Strait Islander women compared to their respective counterparts (Diaz et al., 2015; AIHW, 2017a). Reasons for these disparities include reduced access to screening services leading to later presentation of disease or poorer tumour characteristics, reduced access to appropriate medical services contributing to treatment delays or inability to incomplete treatment, poor health literacy leading to lack of knowledge of breast cancer symptoms, importance of screening and treatment. Furthermore, education and cultural beliefs may contribute to these disparities (Murphy et al., 2015; Ho-Huynh et al., 2019; Malik et al., 2020).

Efforts in Australia during the recent 15-20 years have established resources to reach out to all women, especially those living in rural and remote areas. This may have levelled out the previous disparity between women living in urban centres compared to women living in rural and remote areas. However, this remains to be shown. Hence, the aim of our study was to explore how tumour, patient, and treatment-related factors as well as ethnicity and rurality are associated with survival outcomes for women in an Australian area having both an urban city as well

${ }^{1}$ School of Medicine and Dentistry, James Cook University, Australia Queensland Health, Australia. ${ }^{2}$ Department of Surgery, Cairns and Hinterland Hospital \&amp; Health Service, Queensland Health, Australia. ${ }^{3}$ Primary Health Care, School of Public Health, Institute of Medicine, the Sahlgrenska Academy, University of Gothenburg, Sweden. ${ }^{4}$ Research and Development Primary Health Care, Sweden. ${ }^{5} J a m e s$ Cook University College of Medicine and Dentistry, Australia. *For Correspondence: albert. hohuynh@my.jcu.edu.au 
as areas of rural and remote living. The aim was further specified into the following research questions:

1) What factors are associated with increased risk of breast cancer death for women when first presenting with breast cancer?

2) What are the five, ten, fifteen, and twenty-year survival rates for women when first presenting with breast cancer?

3) Are Aboriginal and Torres Strait Islander status, remoteness of area of residence, and socioeconomic status linked to late presentation of breast cancer (larger tumour size, higher grade tumours, and presence of metastasis in axillary lymph nodes) when first presenting with breast cancer?

\section{Materials and Methods}

A retrospective longitudinal study design was conducted at Cairns Hospital, Queensland, Australia. Cairns Hospital, located in Far North Queensland, is the primary referral centre for the Cape York Peninsula, the Torres Strait Islands and communities surrounding Cairns thus capturing all breast cancers in the well-defined geographical area of regional Far North Queensland. This study was granted ethics approval by the Far North Queensland Human Research Ethics Committee (HREC/17/QCH/121 - 1182). Deidentified data were extracted in July 2018 and analysis was performed using IBM SPSS Version 25.

\section{Inclusion criteria}

All women diagnosed with their first episode of breast cancer at Cairns Hospital, Queensland, Australia were eligible for inclusion in this study for a 15 year period between 1 January 1999 to 31 December 2013. Women not residing within Australia were not included, as death notification would be impractical to follow-up.

\section{Data sources}

A population-based breast cancer dataset created in 1999 of all patients diagnosed with and/or treated for breast cancer at Cairns Hospital was used to extract data. This dataset was complemented with information obtained from paper and electronic hospital medical records and pathology reports. Information pertaining to date, age and cause of death was linked with data from the Queensland Cancer Registry (QCR) for the period of 1 January 1999 to 31 December 2017 to determine the vital status of each woman at a minimum of four years after the date of diagnosis.

\section{Data collected}

The tumour-related factors extracted from the breast cancer dataset include tumour type, tumour size, tumour grade, bilateral synchronous disease, number of tumours, lymphovascular invasion, oestrogen receptor status, progesterone receptor status, HER2 receptor status, and axillary lymph node positivity.

Patient-related factors included age at diagnosis, menopausal status, Aboriginal and Torres Strait Islander status, Remoteness of area of residence, and Socioeconomic status. In the analysis, patients were determined to be Aboriginal and/or Torres Strait Islander if they had identified so on their hospital medical records. Remoteness of area of residence was assigned to each woman based on their locality/suburb at the time of diagnosis. This included major city, inner regional, outer regional, remote, and very remote based on the Australian Standard Geographical Classification Remoteness Structure of the Australian Bureau of Statistics (2011a).

Socioeconomic status was assigned to each woman based on their locality/suburb at the time of diagnosis. This was a percentile value based on the Australian Bureau of Statistics Index of Relative Socioeconomic Disadvantage (IRSD) (2006; 2011b).

Treatment factors were not included as these are individualised for women and any differences in outcomes would reflect more extensive disease at diagnosis (2001a; 2001b). More suitably, treatment refusal (refusal of any part of recommended treatment) was included as a variable for analysis.

\section{Statistical method}

The data was analysed using the Statistical Package for Social Sciences (IBM SPSS) Version 25. For research question one, univariate Cox regression was performed to calculate crude hazard ratios for each of the independent variables collected. Any variable producing a $\mathrm{p}$ value of $<0.2$ was brought forward to the final multivariable analysis, which was predetermined to be done using backwards stepwise multivariable Cox proportional hazard regression. The primary outcome was time to breast cancer death. This analysis was performed twice; once for women with any type of breast cancer (in situ or invasive) and again for women with invasive cancer.

For research question two, five, ten, fifteen, and twenty-year breast cancer-specific absolute survival were estimated for all women presenting with their first episode of breast cancer using life tables. Women dying from other causes were censored.

For research question three, statistical association analysis was performed between demographic factors and late presentation of breast cancer; indicated by strongly significant variables in part one which were associated with more advanced disease (tumour size and axillary node positivity). Standard linear regression was used to assess the association between Aboriginal and Torres Strait Islander status, remoteness area of residence, and socioeconomic status with tumour size. This analysis was performed for women with any type of breast cancer and women with invasive cancer. As tumour size was not normally distributed, Blom's rank transformation was performed on the dependent variable. Binary logistic regression was used to assess the association between Aboriginal and Torres Strait Islander status, remoteness of area of residence, and socioeconomic status with axillary node positivity. This analysis was performed for women with invasive cancer as axillary node positivity was a significant predictor for mortality for these women.

Sample size calculation

A sample size calculation was performed for the 
primary research question using Power Analysis and Sample Size (PASS) Software. Assuming a power of 0.95, an alpha of 0.05 , and hazards ratios of 1.6, 1.4, and 1.3 for Aboriginal and Torres Strait Islander status, remoteness of area of residence, and socioeconomic status respectively, the required sample sizes were 224, 276, and 501 (Hall et al., 2004; Clayforth et al., 2007; Supramaniam et al., 2014). Hence, $>501$ women were required to make the statistical analysis robust.

\section{Results}

A total of 902 patients were diagnosed with breast cancer at Cairns Hospital during the study period 1st of January 1999 up to 31st of December 2013 and 800 of these presented with their first episode of breast cancer. Seventeen patients were excluded from the study including four men, two women residing outside of Australia and eleven duplicates (where women with bilateral disease were entered twice). For the duplicates, data entries which were reflective of more advanced disease were retained. Therefore, 783 women presenting with their first episode of breast cancer at Cairns Hospital were included in the analysis.

The age at diagnosis ranged from 27 to 91 with a mean age of 57 years (Table 1). There was a total of 170 deaths during follow up, of which 100 were identified as breast cancer deaths. The majority of breast cancers were classified as invasive (Table 2).
Survival from Breast Cancer in Rural and Remote Areas

Factors associated with to breast cancer mortality

Increased tumour size and absence of progesterone receptor were associated with increased risk of breast cancer death among women diagnosed with any type of breast cancer (Table 3). For women with invasive cancer increased tumour size, absence of progesterone receptor and presence of metastasis in axillary lymph nodes were associated with increased risk of breast cancer death (Table 4). Invasive tumour grade in women with invasive cancer showed marginal association $(\mathrm{p}=0.049)$ with breast cancer death.

\section{Absolute survival}

The five-, ten-, fifteen- and twenty-year absolute survival rate was found to be $0.90,0.86,0.82$ and 0.80 respectively for all women presenting with their first episode of breast cancer in Far North Queensland between 1999-2013 (Figure 1).

Association between demographic factors and late presentation

For all women, there was no association between Aboriginal and Torres Strait Islander status $(p=0.16)$, remoteness of area of residence $(p=0.51)$, and socioeconomic status $(\mathrm{p}=0.51)$ with larger tumour size. The same was shown for women with invasive cancer with no association between Aboriginal and Torres Strait Islander status $(p=0.12)$, remoteness of area of residence $(p=0.40)$, and socioeconomic status $(p=0.50)$ with larger tumour size. Furthermore, no association was

Table 1. Potential Risk Factors at Diagnosis Measured by a Continuous Scale

\begin{tabular}{lccccc}
\hline Continuous variable & $\mathrm{n}$ & Min & Max & Mean (SD) & Median (IQR) \\
\hline In situ/invasive tumour size $(\mathrm{cm})$ & 736 & 0 & 16 & $2.7(2.4)$ & $2.0(2.3)$ \\
Invasive tumour size $(\mathrm{cm})$ & 662 & 0 & 16 & $2.7(2.3)$ & $2.0(2.3)$ \\
Age at diagnosis & 783 & 27 & 91 & $57(12)$ & $56(17)$ \\
Socioeconomic status* & 752 & 1 & 94 & $34(26)$ & $27(40)$ \\
\hline * Socioeconomic status is measured as a percentile, with lowest socioeconomic status being 1 and highest being 100. The Australian average is 50.
\end{tabular}

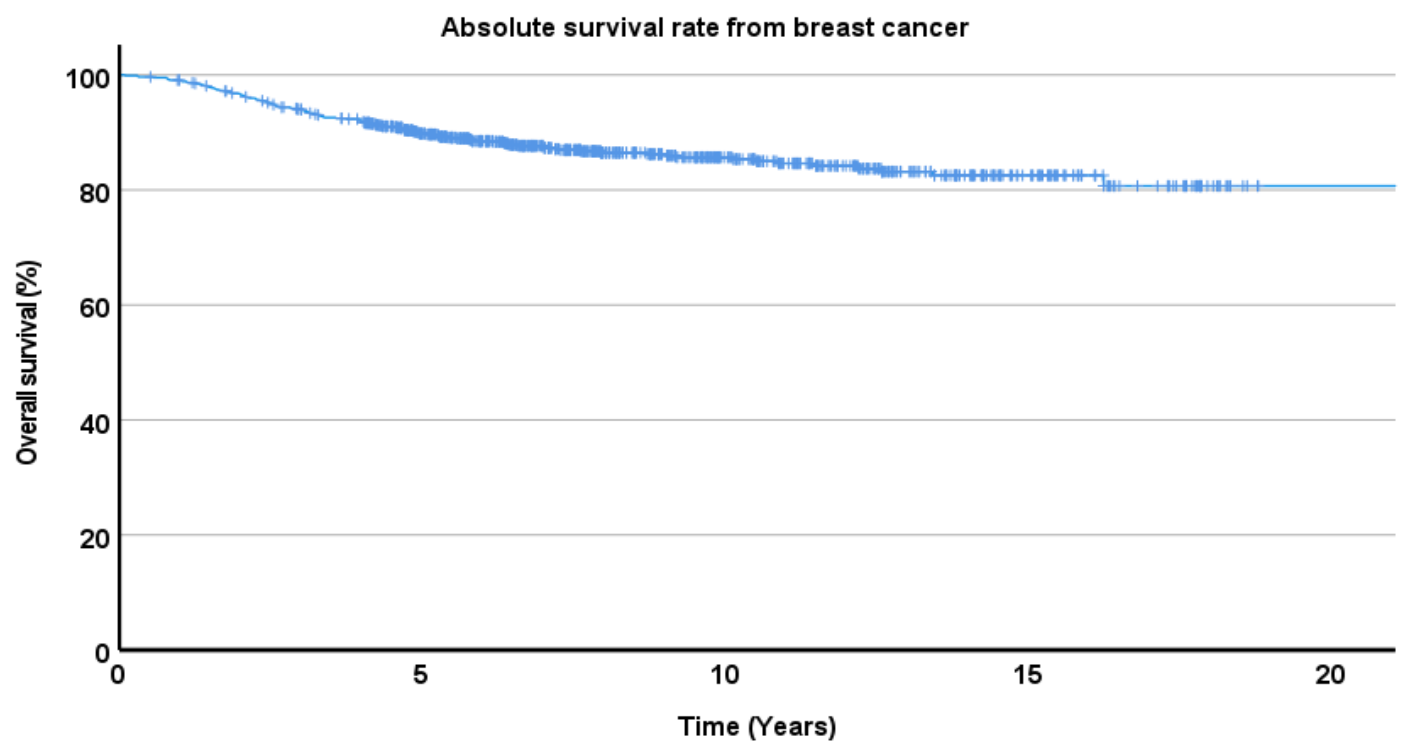

Figure 1. Absolute Survival from Breast Cancer for All Women Presenting with Their First Episode of Breast Cancer between 1999-2013 
Table 2. Potential Risk Factors at Diagnosis Measured by a Nominal or Ordinal Scale

\begin{tabular}{|c|c|}
\hline Categorical variables & $\mathrm{n}(\%)$ \\
\hline \multicolumn{2}{|l|}{ Tumour type } \\
\hline In situ & $73 / 779(9.4)$ \\
\hline Invasive & $706 / 779(91)$ \\
\hline \multicolumn{2}{|l|}{ Invasive tumour type } \\
\hline $\begin{array}{l}\text { Invasive lobular, Invasive cancer } \\
\text { specified, Other }\end{array}$ & $100 / 682(15)$ \\
\hline Ductal NOS & $582 / 682(85)$ \\
\hline \multicolumn{2}{|l|}{ Invasive tumour grade } \\
\hline Grade 1 & $165 / 670(25)$ \\
\hline Grade 2 & $293 / 670(44)$ \\
\hline Grade 3 & $209 / 670(31)$ \\
\hline Malignant & $3 / 670(0.45)$ \\
\hline \multicolumn{2}{|l|}{ Bilateral synchronous disease } \\
\hline No & $765 / 783(98)$ \\
\hline Yes & $18 / 783(2.3)$ \\
\hline \multicolumn{2}{|l|}{ Number of tumours } \\
\hline One & $621 / 736(84)$ \\
\hline Two or more & $115 / 736(16)$ \\
\hline \multicolumn{2}{|l|}{ Lymphovascular invasion } \\
\hline Absent & $519 / 718(72)$ \\
\hline Present & $199 / 718(28)$ \\
\hline \multicolumn{2}{|l|}{ Axillary node positivity } \\
\hline Negative & $497 / 729(68)$ \\
\hline Positive & $232 / 729(32)$ \\
\hline \multicolumn{2}{|l|}{ Oestrogen receptor } \\
\hline Negative & $171 / 750(23)$ \\
\hline Positive & $579 / 750(77)$ \\
\hline \multicolumn{2}{|l|}{ Progesterone receptor } \\
\hline Negative & $204 / 653(31)$ \\
\hline Positive & $449 / 653(69)$ \\
\hline \multicolumn{2}{|l|}{ HER2 receptor } \\
\hline Negative & $450 / 582(77)$ \\
\hline Positive & $132 / 582(23)$ \\
\hline \multicolumn{2}{|l|}{ Premenopausal } \\
\hline No & $465 / 781(60)$ \\
\hline Yes & $316 / 781(40)$ \\
\hline \multicolumn{2}{|l|}{ Aboriginal and Torres Strait Islander status } \\
\hline Non-indigenous & $669 / 768(87)$ \\
\hline $\begin{array}{l}\text { Aboriginal, Torres Strait Islander, Both } \\
\text { Aboriginal and Torres Strait Islander }\end{array}$ & $99 / 768(13)$ \\
\hline \multicolumn{2}{|l|}{ Remoteness of area of residence } \\
\hline Major city, inner regional, outer regional & $711 / 783(91)$ \\
\hline Remote, very remote & $72 / 783(9.2)$ \\
\hline \multicolumn{2}{|l|}{ Treatment refusal } \\
\hline No & $602 / 783(77)$ \\
\hline Yes & $181 / 783(23)$ \\
\hline
\end{tabular}

demonstrated for Aboriginal and Torres Strait Islander status $(p=0.08)$, remoteness of area of residence $(p=0.83)$, and socioeconomic status $(\mathrm{p}=0.76)$ with axillary node positivity. Hence, this study could not demonstrate any association between demographic factors and more advanced disease at presentation.

\section{Discussion}

Poorer breast cancer outcomes has been reported for women living in rural areas, women of lower socioeconomic status, and Aboriginal and Torres Strait Islander women.(Youlden et al., 2012; AIHW, 2017a) Our study in Far North Queensland challenges these findings and demonstrate that regional areas that are well resourced with effective infrastructure and strategies in place can achieve similar outcomes to major cities.(Murphy et al., 2015; Platt et al., 2015)

\section{Rurality and remoteness}

Studies in many developed countries, including Australia, have reported that Aboriginal and Torres Strait Islander women, women living in rural areas, and women of low socioeconomic status often present with more advanced disease (Clayforth et al., 2007; Downing et al., 2007; Curtis et al., 2008; Azzopardi et al., 2014; AIHW, 2017a). However, in this study there was no proven association between these specific demographic factors with more advanced disease. Furthermore, the five-year absolute survival in Far North Queensland was 90\% which, compared to Australia nationally with a five-year relative survival of $90 \%$ is equivalent if not better (AIHW, 2017a). The lack of difference between these groups of women may be because national efforts to reduce these disparities in the past are now being reflected in the results of this study showing that the gap in previous inequalities has closed.

While population screening programs, such as BreastScreen Australia, are effective in detecting asymptomatic and early disease, further breast cancer awareness and public campaigns may contribute to increasing participation rates in screening and targeting groups of women who are more likely to present with more advanced disease (AIHW, 2017b). Education is most likely the key to success in countries where the level of education among women is very low. (Malik et al., 2020)

\section{Culturally sensitive strategies for closing the gap}

Multidisciplinary team meetings, telehealth, regional cancer strategic meetings, web-based information, cancer nurse co-ordinators, and new models of care combined have been proven to be effective strategies in addressing barriers in access to high standard healthcare and consequently closing the gap in inequality (Platt et al., 2015).

Population mammography screening programs not only help to detect early breast cancer but also improve survival outcomes (Jatoi and Miller, 2003). The extensive regional coverage of BreastScreen Queensland with service locations in Cairns and relocatable breast cancer screening units to rural areas across Far North Queensland has resulted in greater participation of women in breast 
DOI:10.31557/APJCP.2022.23.1.101

Survival from Breast Cancer in Rural and Remote Areas

Table 3. Factors Associated with Breast Cancer Mortality for Women Diagnosed with Their First Episode of Any Type of Breast Cancer (in Situ or Invasive).

\begin{tabular}{|c|c|c|c|c|c|}
\hline \multirow[b]{2}{*}{ Independent variables } & \multirow[b]{2}{*}{$\mathrm{n}$} & \multicolumn{2}{|c|}{ Unadjusted } & \multicolumn{2}{|c|}{ Adjusted $(\mathrm{n}=531)$} \\
\hline & & $\mathrm{p}$ value & $\mathrm{HR}$ & $\mathrm{p}$ value & HR \\
\hline Tumour type & 779 & 0.027 & $24(1.4-4.0 \times 102)$ & & \\
\hline Tumour size $\mathrm{cm}$ & 736 & $2.6 \times 10^{-8}$ & $1.2(1.1-1.2)$ & 0.017 & $1.1(1.0-1.2)$ \\
\hline Bilateral synchronous disease & 783 & 0.37 & $1.6(0.58-4.3)$ & & \\
\hline Number of tumours & 736 & 0.63 & $0.86(0.47-1.6)$ & & \\
\hline Lymphvascular invasion & 718 & $8.4 \times 10^{-11}$ & $4.6(2.9-7.2)$ & 0.08 & $2.0(0.92-4.2)$ \\
\hline Oestrogen receptor & 750 & 0.0032 & $0.52(0.34-.80)$ & & \\
\hline Progesterone receptor & 653 & 0.000065 & $0.39(0.24-.62)$ & 0.0011 & $0.39(0.23-.69)$ \\
\hline HER2 receptor & 582 & 0.064 & $1.6(0.97-2.7)$ & & \\
\hline Axillary node positivity & 729 & $8.1 \times 10^{-9}$ & $3.8(2.4-5.9)$ & 0.059 & $2.1(0.97-4.6)$ \\
\hline Age at diagnosis & 783 & 0.39 & $0.99(0.98-1.0)$ & & \\
\hline Premenopausal & 781 & 0.089 & $1.4(0.95-2.1)$ & & \\
\hline Aboriginal and Torres Strait Islander status & 768 & 0.15 & $1.5(0.87-2.5)$ & & \\
\hline Remoteness of area of residence & 783 & 0.47 & $0.76(0.35-1.6)$ & & \\
\hline Socioeconomic status & 752 & 0.86 & $1.0(0.99-1.0)$ & & \\
\hline Treatment refusal & 783 & 0.11 & $1.4(0.93-2.2)$ & & \\
\hline
\end{tabular}

screening and subsequent improvement in survival (Jatoi and Miller, 2003; Youlden et al., 2012; AIHW, 2017b).

Participation rate in BreastScreen in Northern Queensland has been found to be consistently the highest, at a rate of $63 \%$ compared to the national participation rate of 54\% (AIHW, 2018). Furthermore, culturally sensitive cancer services and projects aimed at removing the stigma of cancer in Aboriginal communities have led to larger numbers of Aboriginal and Torres Strait Islander women participating in cancer screening programs and treatment.(Flegg et al., 2010; Diaz et al., 2015) Programs such as these, including "Closing the Gap on Breast Cancer Screening" and "Out in the Open", and involving Indigenous liaison officers and Aboriginal health workers have helped identify breast cancer in Aboriginal and Torres Strait Islander women who would have otherwise

Table 4. Factors Associated with Breast Cancer Mortality for Women Diagnosed with Their First Episode of Invasive Breast Cancer

\begin{tabular}{|c|c|c|c|c|c|}
\hline \multirow[b]{2}{*}{ Independent variables } & \multirow[b]{2}{*}{$\mathrm{n}$} & \multicolumn{2}{|c|}{ Unadjusted } & \multicolumn{2}{|c|}{ Adjusted $(\mathrm{n}=514)$} \\
\hline & & $\mathrm{p}$ value & HR & $\mathrm{p}$ value & HR \\
\hline Invasive tumour type & 682 & 0.068 & $2.1(.95-4.4)$ & & \\
\hline Invasive tumour grade & 670 & -- & & & \\
\hline Grade 1 & & 0.000003 & Ref & 0.131 & Ref \\
\hline Grade 2 & & 0.0025 & $6.2(1.9-20)$ & 0.053 & $3.3(0.99-11)$ \\
\hline Grade 3 & & 0.000011 & $14(4.3-44)$ & 0.049 & $3.6(1.0-13)$ \\
\hline Malignant & & 0.0068 & $23(2.4-220)$ & $-* *$ & $-* *$ \\
\hline Invasive tumour size $(\mathrm{cm})$ & 662 & $5.6 \times 10^{-10}$ & $1.2(1.1-1.3)$ & 0.01 & $1.1(1.0-1.2)$ \\
\hline Bilateral synchronous disease & 706 & 0.5 & $1.4(.52-3.8)$ & & \\
\hline Number of tumours & 664 & 0.906 & $0.96(.52-1.8)$ & & \\
\hline Lymphovascular invasion & 644 & $6.7 \times 10^{-9}$ & $3.9(2.5-6.2)$ & & \\
\hline Oestrogen receptor & 682 & 0.0021 & $0.50(.33-.78)$ & & \\
\hline Progesterone receptor & 593 & 0.0001 & $0.40(.25-.63)$ & 0.013 & $0.47(0.26-.85)$ \\
\hline HER2 receptor & 567 & 0.06 & $1.6(.98-2.7)$ & & \\
\hline Axillary node positivity & 656 & $4.9 \times 10^{-7}$ & $3.2(2.0-5.0)$ & 0.0023 & $2.7(1.4-5.1)$ \\
\hline Age at diagnosis & 706 & 0.43 & $0.99(.98-1.0)$ & & \\
\hline Premenopausal & 704 & 0.1 & $1.4(.94-2.1)$ & & \\
\hline Aboriginal and Torres Strait Islander status & 693 & 0.16 & $1.5(.86-2.5)$ & & \\
\hline Remoteness of area of residence & 706 & 0.51 & $0.77(.36-1.7)$ & & \\
\hline Socioeconomic status & 676 & 0.71 & $1.0(.99-1.0)$ & & \\
\hline Treatment refusal & 706 & 0.17 & $1.4(.88-2.1)$ & 0.085 & $1.7(0.93-3.1)$ \\
\hline
\end{tabular}


presented with advanced disease and the associated poorer outcomes (Roder et al., 2012; AIHW, 2017b)

Additionally, the introduction of breast care nurses at Cairns Hospital in 2002 may have further contributed to better breast cancer outcomes both physically and psychologically by coordinating cancer care and providing individualised information and emotional support to patients and their families in a critical time in their lives (Eicher et al., 2006). This is further supported by previous studies demonstrating that breast cancer patients, particularly in rural and remote areas, recognise the importance of breast care nurses and are highly satisfied with the level of care breast care nurses provide (Eley et al., 2008). Improving access to breast care nurses and involving them throughout diagnosis to treatment shows great promise for reducing disparities in breast cancer outcomes, particularly in populations where poorer outcomes are reported.

\section{Strengths and limitations}

A great advantage of this study is that it is the first breast cancer survival study in regional Far North Queensland that accounts for disparities in breast cancer survival by adjusting for differences in tumour-, patient-, and treatment-related factors. Another strength of this study is the adequacy of sample size based on thorough sample-size calculations.

The data collected are based on a combination of paper and electronic data sets which are subject to human error and missing data. Consequently, there is the possibility of selection bias due to the exclusion of patients with missing data in the statistical analysis. This included $17 \%$ and $25 \%$ of missing data for the progesterone and HER2 receptor respectively as pathological confirmation of these receptors were not routinely performed in the pathology lab until the mid-2000s. Despite this, the data is the most complete to date with less than $8 \%$ of missing data across any other variable. Lastly, due to the unavailability of population life tables, relative survival could not be calculated.

There are different statistical techniques to select independent variables into a multivariable model. This study does not aim to construct a precise prediction model but rather to get a brief estimate of the relative importance of demographic factors such as rurality, socioeconomic standard and ethnicity versus traditional risk factors. Hence, stepwise regression, presented also with unadjusted regressions, was deemed sufficient.

In conclusion, this study suggests that given appropriate resources it may be possible to close the gap of inequalities in breast cancer. However, this study reflects the situation in Far North Queensland Australia where substantial efforts have been made in closing the gap of inequalities and inequalities may well remain elsewhere. This study should be taken as an encouragement to further investigate inequalities elsewhere and, when found address them.

\section{Author Contribution Statement}

$\mathrm{AH}, \mathrm{TE}, \mathrm{RG}, \mathrm{AdC}$ were all involved in project proposal and planning. AH and TE performed data collection. AH and RG analysed and interpreted the data set. AH prepared the final manuscript. All authors read and approved the final manuscript. The datasets generated during and analysed during the current study are not publicly available due the terms and conditions of the ethics application but are available from the corresponding author on reasonable request

\section{Acknowledgements}

This study was undertaken with the ethics approval of the Far North Queensland Human Research Ethics Committee (HREC/17/QCH/121 - 1182) and funded by a grant from James Cook University, School of Medicine and Dentistry (Reference number JCU-QLD-665491). The study sponsors had no involvement in any of the study design, collection, analysis and interpretation of data; in the writing of the manuscript; and in the decision to submit the manuscript for publication.

\section{Conflicts of interest}

There are no conflicts of interest to declare.

\section{References}

2001a. National Breast Cancer Centre. Clinical practice guidelines: management of advanced breast cancer, Canberra, Commonwealth of Australia.

2001b. National Breast Cancer Centre. Clinical practice guidelines: management of early breast cancer, Canberra, Commonwealth of Australia.

AIHW. 2017a. Australian Cancer Incidence and Mortality (ACIM) books: Breast cancer. [Online]. Australian Institute of Health and Welfare. Available: www.aihw.gov.au/acimbooks

Azzopardi J, Walsh D, Chong C, et al (2014). Impact of geographic location on surgical outcomes of women with breast cancer. Aust N Z J Surg, 84, 735-9.

Clayforth C, Fritschi L, McEvoy SP, et al (2007). Five-year survival from breast cancer in Western Australia over a decade. Breast J, 16, 375-81.

Curtis E, Quale C, Haggstrom D, et al (2008). Racial and Ethnic Differences in Breast Cancer Survival: How much is explained by screening, tumor severity, biology, treatment, comorbidities, and demographics?. Cancer J, 112, 171-80.

Diaz A, Whop LJ, Valery PC, et al (2015). Cancer outcomes for Aboriginal and Torres Strait Islander Australians in rural and remote areas. Aust J Rural Health, 23, 4-18.

Downing A, Prakash K, Gilthorpe MS, et al (2007). Socioeconomic background in relation to stage at diagnosis, treatment and survival in women with breast cancer. $\mathrm{Br} J$ Cancer, 96, 836-40.

Eicher MR, Marquard S, Aebi S (2006). A nurse is a nurse? A systematic review of the effectiveness of specialised nursing in breast cancer. Eur J Cancer, 42, 3117-26.

Eley RM, Rogers-Clark C, Murray K (2008). The value of a breast care nurse in supporting rural and remote cancer patients in Queensland. Cancer Nurs, 31, E10-8.

Flegg KM, Flaherty JJ, Bicknell AM, et al (2010). Surgical outcomes of borderline breast lesions detected by needle biopsy in a breast screening program. World J Surg Oncol, 8.

Ghoncheh M, Pournamdar Z, Salehiniya H (2016). Incidence and mortality and epidemiology of breast cancer in the world. Asian Pac J Cancer Prev, 17, 43-6.

Hall S, Holman CD, Sheiner H, et al (2004). The influence of 
socio-economic and locational disadvantage on survival after a diagnosis of lung or breast cancer in Western Australia. $J$ Health Services Research Policy, 9, 2, 10-S2:6.

Ho-Huynh A, Tran A, Bray G, et al (2019). Factors influencing breast cancer outcomes in Australia: A systematic review. Eur J Cancer Care (Engl), 28, e13038.

Jatoi I, Miller AB (2003). Why is breast-cancer mortality declining?. Lancet Oncol, 4, 251-4.

Malik R, Vera N, Dayal C, et al (2020). Factors associated with breast cancer awareness and breast self-examination in Fiji and Kashmir India - a cross-sectional study. BMC Cancer, 20, 1078 .

McLafferty S, Wang F (2009). Rural reversal? Rural-urban disparities in late-stage cancer risk in Illinois. Cancer, 115, 2755-64.

Murphy C, Sabesan S, Steer C, et al (2015). Oncology service initiatives and research in regional Australia. Aust J Rural Health, 23, 40-8.

Platt V, O'Connor K, Coleman R (2015). Improving regional and rural cancer services in Western Australia. Aust J Rural Health, 23, 32-9.

Roder D, Webster F, Zorbas H, et al (2012). Breast screening and breast cancer survival in Aboriginal and Torres Strait Islander women of Australia. Asian Pac J Cancer Prev, 13, 147-55.

Supramaniam R, Gibberd A, Dillon A, et al (2014). Increasing rates of surgical treatment and preventing comorbidities may increase breast cancer survival for Aboriginal women. BMC Cancer, 14.

Youlden DR, Cramb SM, Dunn NA, et al (2012). The descriptive epidemiology of female breast cancer: an international comparison of screening, incidence, survival and mortality. Cancer Epidemiol, 36, 237-48.

\section{(c) (1) (3)}

This work is licensed under a Creative Commons AttributionNon Commercial 4.0 International License. 